\title{
CONNES-AMENABILITY AND NORMAL, VIRTUAL DIAGONALS FOR MEASURE ALGEBRAS, II
}

\section{VOLKER RUNDE}

We prove that the following are equivalent for a locally compact group $G$ :

(i) $G$ is amenable;

(ii) $M(G)$ is Connes-amenable;

(iii) $M(G)$ has a normal, virtual diagonal.

\section{THE RESULT}

A Banach algebra $\mathfrak{A}$ is called dual if it is a dual space such that multiplication in $\mathfrak{A}$ is separately $w^{*}$-continuous. A Banach bimodule over a dual Banach algebra is called normal if it is a dual space such that the module operations are separately $w^{*}$-continuous (see $[6,7,8]$ ).

Definition 1: A dual Banach algebra $\mathfrak{A}$ is called Connes-amenable if, for every normal Banach $\mathfrak{A}$-bimodule $E$, every $w^{*}$-continuous derivation $D: \mathfrak{A} \rightarrow E$ is inner.

The notion of Connes-amenability was introduced for von Neumann algebras in [5] (the name "Connes-amenability" seems to originate from [3]); it is equivalent to a number of important von Neumann algebraic properties such as injectivity, semidiscreteness, and being approximately finite-dimensional (see [7, Chapter 6] for a self-contained exposition and references to the original literature).

For arbitrary dual Banach algebras, Connes-amenability was first considered in [6], and in [8] it was shown that $M(G)$, the measure algebra of a locally compact group $G$, is Connes-amenable if and only if $G$ is amenable.

For any dual Banach algebra $\mathfrak{A}$, let $\mathcal{L}_{w^{*}}^{2}(\mathfrak{A}, \mathbb{C})$ denote the separately $w^{*}$-continuous bilinear functionals on $\mathfrak{A}$. It is easy to see that the multiplication map $\Delta: \mathfrak{A} \otimes \mathfrak{A} \rightarrow \mathfrak{A}$ extends to $\mathcal{L}_{w^{*}}^{2}(\mathfrak{A}, \mathbb{C})^{*}$ as a continuous $\mathfrak{A}$-bimodule homomorphism.

Definition 2: Let $\mathfrak{A}$ be a dual Banach algebra. A normal, virtual diagonal for $\mathfrak{A}$ is an element $\mathrm{M} \in \mathcal{L}_{w^{*}}^{2}(\mathfrak{A}, \mathbb{C})^{*}$ such that

$$
a \cdot \mathrm{M}=\mathrm{M} \cdot a \quad \text { and } \quad a \Delta \mathrm{M}=a \quad(a \in \mathfrak{A}) .
$$

Received 18th March, 2003

Research supported by NSERC under grant no. 227043-00.

Copyright Clearance Centre, Inc. Serial-fee code: 0004-9727/03 \$A2.00+0.00. 
If $\mathfrak{A}$ has a normal, virtual diagonal, then it is Connes-amenable $([1,2])$. The converse holds if $\mathfrak{A}$ is a von Neumann algebra ([2]). It is an open question - likely with a negative answer - if Connes-amenability and the existence of normal, virtual diagonals are equivalent for arbitrary dual Banach algebras.

In [6] and [8], we gave partial positive answers for $\mathfrak{A}=M(G)$ :

1. If $G$ is compact, then $M(G)$ has a normal, virtual diagonal ([6, Proposition 5.2] and [8, Proposition 3.3]).

2. If $G$ is discrete, then $M(G)=\ell^{1}(G)$ is Connes-amenable if and only if it has a normal, virtual diagonal ([8, Corollary 5.4]).

In this note, we shall prove the following theorem, and thus extend [8, Theorem 5.3].

THEOREM 1. The following are equivalent for a locally compact group $G$ :

(i) $G$ is amenable.

(ii) $M(G)$ is Connes-amenable.

(iii) $M(G)$ has a normal, virtual diagonal.

\section{THE PROOF}

For convenience, we quote the following well-known characterisation of amenable locally compact groups (see [7, Lemma 7.1.1], for example).

Lemma 1. A locally compact group $G$ is amenable if and only if there is a net $\left(f_{\alpha}\right)_{\alpha}$ of non-negative functions in the unit sphere of $L^{1}(G)$ such that

$$
\sup _{x \in K}\left\|\delta_{x} * f_{\alpha}-f_{\alpha}\right\| \rightarrow 0
$$

for each compact subset $K$ of $G$.

Proof of THE ThEOREM: In view of [8, Theorem 5.3], it is sufficient to show that (i) implies (iii).

Let $\left(f_{\alpha}\right)_{\alpha}$ be a net as specified in the lemma. Define a net $\left(m_{\alpha}\right)_{\alpha}$ in $M(G \times G)$ by letting

$$
\left\langle f, m_{\alpha}\right\rangle:=\int_{G} f\left(x, x^{-1}\right) f_{\alpha}(x) d x \quad\left(f \in \mathcal{C}_{0}(G \times G)\right),
$$

where $d x$ denotes integration with respect to left Haar measure on $G$. Let $y \in G$, and note that, for $f \in \mathcal{C}_{0}(G \times G)$,

$$
\begin{aligned}
\left\langle f,\left(\delta_{y} \otimes \delta_{e}\right) * m_{\alpha}\right\rangle & =\int_{G} f\left(y x, x^{-1}\right) f_{\alpha}(x) d x \\
& =\int_{G} f\left(x, x^{-1} y\right) f_{\alpha}\left(y^{-1} x\right) d x \quad \text { (substitute } y^{-1} x \text { for } x \text { ) } \\
& =\int_{G} f\left(x, x^{-1} y\right)\left(\delta_{y} * f_{\alpha}\right)(x) d x
\end{aligned}
$$


and

$$
\left\langle f, m_{\alpha} *\left(\delta_{e} \otimes \delta_{y}\right)\right\rangle=\int_{G} f\left(x, x^{-1} y\right) f_{\alpha}(x) d x .
$$

It follows from $(*)$ that

$$
\sup _{y \in K}\left\|\left(\delta_{y} \otimes \delta_{e}\right) * m_{\alpha}-m_{\alpha} *\left(\delta_{e} \otimes \delta_{y}\right)\right\| \rightarrow 0
$$

for each compact subset $K$ of $G$.

By $\left[8\right.$, Proposition 3.1], we may identify the Banach $M(G)$-bimodules $\mathcal{L}_{w^{*}}^{2}(M(G), \mathbb{C})$ and

$$
\mathcal{S C}_{0}(G \times G):=\left\{f \in \ell^{\infty}(G \times G): f(\cdot, x), f(x, \cdot) \in \mathcal{C}_{0}(G) \text { for each } x \in G\right\}
$$

Let $\mathcal{U}$ be an ultrafilter on the index set of $\left(m_{\alpha}\right)_{\alpha}$ that dominates the order filter. Define $\mathrm{M} \in \mathcal{S C}_{0}(G \times G)^{*}$ by letting

$$
\langle f, \mathrm{M}\rangle:=\lim _{\mathcal{U}} \int_{G \times G} f(x, y) d m_{\alpha}(x, y) \quad\left(f \in \mathcal{S C}_{0}(G \times G)\right)
$$

(since all functions in $\mathcal{S C}_{0}(G \times G)$ are measurable with respect to any Borel measure by [4], the integrals do exist). It is routinely seen that $\Delta \mathrm{M}=\delta_{e}$.

Let $\mu \in M(G)$ and let $f \in \mathcal{S C}_{0}(G \times G)$. Then we have:

$$
\begin{aligned}
\mid\langle f, \mu & \cdot \mathrm{M}-\mathrm{M} \cdot \mu\rangle \mid \\
& =|\langle f \cdot \mu-\mu \cdot f, \mathrm{M}\rangle| \\
& =\left|\lim _{\mathcal{U}} \int_{G \times G}\left(\int_{G}(f(z x, y)-f(x, y z)) d \mu(z)\right) d m_{\alpha}(x, y)\right| \\
& =\left|\lim _{\mathcal{U}} \int_{G}\left(\int_{G \times G}(f(z x, y)-f(x, y z)) d m_{\alpha}(x, y)\right) d \mu(z)\right| \quad \text { (by Fubini's theorem) } \\
& \leqslant \lim _{\mathcal{U}} \int_{G}\left|\int_{G \times G}(f(z x, y)-f(x, y z)) d m_{\alpha}(x, y)\right| d|\mu|(z) \\
& =\lim _{\mathcal{U}} \int_{G}\left|\int_{G \times G} f(x, y) d\left(\left(\delta_{z} \otimes \delta_{e}\right) * m_{\alpha}-m_{\alpha} *\left(\delta_{e} \otimes \delta_{z}\right)\right)(x, y)\right| d|\mu|(z) \\
& \leqslant \lim _{\mathcal{U}} \int_{G}\|f\|\left\|\left(\delta_{z} \otimes \delta_{e}\right) * m_{\alpha}-m_{\alpha} *\left(\delta_{e} \otimes \delta_{z}\right)\right\| d|\mu|(z) \\
& \rightarrow 0 \quad(\text { by }(* *) \text { and the inner regularity of }|\mu|) .
\end{aligned}
$$

It follows that $\mathrm{M}$ is a normal, virtual diagonal for $M(G)$.

\section{REFERENCES}

[1] G. Corach and J.E. Galé, 'Averaging with virtual diagonals and geometry of representations', in Banach algebras '97, (E. Albrecht and M. Mathieu, Editors) (Walter de Grutyer, Berlin, 1998), pp. 87-100. 
[2] E.G. Effros, 'Amenability and virtual diagonals for von Neumann algebras', J. Funct. Anal. 78 (1988), 137-153.

[3] A.Ya. Helemskiǐ, 'Homological essence of amenability in the sense of A. Connes: the injectivity of the predual bimodule', (translated from Russion), Math. USSR-Sb 68 (1991), 555-566.

[4] B.E. Johnson, 'Separate continuity and measurability', Proc. Amer. Math. Soc. 20 (1969), 420-422.

[5] B.E. Johson, R.V. Kadison and J. Ringrose, 'Cohomology of operator algebras, III', Bull. Soc. Math. France 100 (1972), 73-79.

[6] V. Runde, 'Amenability for dual Banach algebras', Studia Math. 148 (2001), 47-66.

[7] V Runde, Lectures on amenability, Lecture Notes in Mathematics 1774 (Springer Verlag, Berlin, Heidelberg, New York, 2002).

[8] V. Runde, 'Connes-amenability and normal, virtual diagonals for measure algebras', $J$. London Math. Soc. 67 (2003), 643-656.

Department of Mathematical and Statistical Sciences

University of Alberta

Edmonton, Alberta

Canada T6G 2G1

e-mail: vrunde@ualberta.ca

URL: http://ww.math.ualberta.ca/ $\sim$ runde 